\title{
Improved diagnosis of tuberculous pleural effusion by combining medical thoracoscopy with Interferon-Gamma Release Assay and adenosine deaminase activity
}

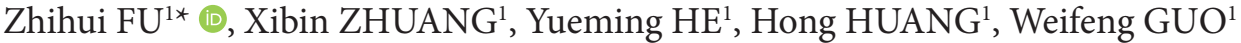

\begin{abstract}
Rapid, accurate, affordable, point-of-care tuberculosis (TB) diagnostic tests are essential for controlling TB. We aimed evaluate the diagnostic performance of tuberculous pleural effusion by combining medical thoracoscopy with blood interferon-gamma release assay (IGRA) and pleural fluid adenosine deaminase (ADA). Patients with undiagnosed pleural effusion measured by chest X-ray or ultrasound were prospectively enrolled in this study. Medical thoracoscopy, blood IGRA and pleural fluid ADA were conducted.A total of 154 patients with undiagnosed pleural effusion were enrolled. Among them, 98 patients (63.6\%) were diagnosed as TPE. Patients in TPE+ groups were significantly younger. The diagnostic thresholds obtained via receiver operating characteristic curve analysis were: ADA $23.4 \mathrm{U} / \mathrm{L}$ with AUC 0.91 (95\% CI: 0.85-0.97, Figure 2) and IFN- $\gamma 6.9 \mathrm{pg} / \mathrm{mL}$ with AUC 0.87 (95\% CI: $0.82-0.93$ ). By combining all three test together, we achieved sensitivity of 0.92 , perfect specificity and NLR of 0.082. The AUC of the combination test was 0.96 (0.93-0.99), which was significant higher than any individual test $(\mathrm{p}<0.001)$. The combination of medical thoracoscopy with Interferon-Gamma release assay and adenosine deaminase performs better than individual test in diagnosis TPE. The combination diagnosis has very high diagnostic rate, can be easily and safely carried out.
\end{abstract}

Keywords: combination test; diagnosis; tuberculous pleural effusion; medial thoracoscopy.

Practical Application: the diagnosis and treatment of a rare and severe disorder.

\section{Introduction}

Tuberculosis (TB) has long plagued human beings and was declared a global emergency in 1993 by WHO (World Health Organization, 1993). Although the incidence and mortality of TB declined over time, there were still about 8.6 million new cases of TB and 1.3 million deaths attributed to TB worldwide in 2012 (World Health Organization, 2013). Although TB is a major public health problem in developing countries, especially in Asia and Africa, TB continues to be of concern in high-income nations (Pareek et al., 2016). In developing countries, a lot of changes in these countries actually increase TB risk, such as rapid urbanization with high population densities and increased rates of cigarette smoking (Abegunde et al., 2007). In high-income countries, the overall changes in TB showed an important disparity, especially in foreign-born immigrants: in some developed countries, foreign-born cases has even increased (Jelastopulu et al., 2009). Additionally, drug-resistant TB emerged as one of the main challenges: about 630,000 cases of multidrug-resistant (MDR) TB occur worldwide, and extensively drug-resistant (XDR) was reported in more than 80 countries (World Health Organization, 2013). On top of the aforementioned challenges, nowadays, insufficient rapid and accurate $\mathrm{TB}$ diagnostic test remain one essential barrier to global TB control activities (Zumla et al., 2013). Rapid, accurate, affordable, point-of-care TB diagnostic tests that are easy to use and implement are needed.
Tuberculous pleural effusion (TPE) is the second most common form of extrapulmonary TB and is the most common cause of pleural effusion in areas where tuberculosis is endemic (Zhai et al., 2016; Light, 2010). The prevalence of pleural effusions among patients with TB varied significantly across different countries. More than 25\% of patient with TB have TPE in Burundi (Mlika-Cabanne et al., 1995). In South Africa, about $20 \%$ of TB patients were diagnosed with TPE (Saks \& Posner, 1992). While in the United States, only 3-5\% of patients with TB were observed to have TPE (Mehta et al., 1991). In addition, immunocompromised patients are more likely to have pleural effusions. The prevalence of pleural effusion was reported higher in HIV-positive patients than immunocompetent patients in South Africa (38\% versus 20\%), Zimbabwe (27\% vs $13 \%$ ), and Uganda (23\% vs 11\%) (Light, 2010). Without treatment, tuberculous pleuritic might resolve spontaneously, but patients frequently develops active TB at follow-up. In a study following 2816 Finnish Armed forces member for at least 7 years, $43 \%$ of patients with pleural effusion later developed TB (Patiala, 1954).

Nowadays, no formal guidelines are available for diagnosis and treatment of tuberculous pleurisy. The gold standard diagnosis of tuberculous pleurisy is the demonstration of tubercle bacilli in the sputum, pleural fluid, or pleural biopsy specimens, or the demonstration of granulomas in the pleural biopsy (Gopi et al., 2007). However, detecting mycobacteria in pleural fluid can be difficult because there may be a large volume of fluid 
and very low numbers of bacteria present. Elevated levels of adenosine deaminase (ADA) or $\gamma$-interferon release assay (IGRA) in the pleural fluid can be treated as a presumptive diagnosis (Trajman et al., 2008). The present data analysis suggests that pleural fluid ADA has good sensitivity (0.92) and specificity (0.90) for diagnosing tuberculous pleuritic (Salmanzadeh et al., 2015). However, ADA also elevated in complicated parapneumonic effusions, emphyemas and lymphomas (Porcel et al., 2010). In addition, its diagnostic utility varies considerably across different geographical regions, clinical settings and test result thresholds (Salmanzadeh et al., 2015). Even though the most widely accepted threshold ADA value is $35-40 \mathrm{U} / \mathrm{L}$, some studies suggested lower cutoffs should be considered in elderly population since ADA decreases with age (Uskul et al., 2005).

Medical thoracoscopy is a safe and efficient method to further differential diagnosis of tuberculous pleurisy (Wang et al., 2015b). However, only a few studies have assessed its applications in pleural effusions (Sakuraba et al., 2006; Wang et al., 2015a), and no study has tested the diagnostic performance of medical thoracoscopy combining with IGRA and ADA. In this study, we aimed to evaluate the diagnostic performance of TPE by combining medical thoracoscopy with IGRA and ADA.

\section{Methods}

\subsection{Patients}

This prospective study followed the principles outlined in the Declaration of Helsinki and was approved by the Institutional Review Board of our Hospital. Written informed consent was signed by all patients. All patients admitted to the Respiratory Department from October 2016 to October 2018 were screened. Patients with undiagnosed pleural effusion were enrolled. Pleural effusion was measured by chest X-ray or ultrasound. All patients received medical thoracoscopy, blood IGRA, and pleural fluid ADA test.

\subsection{Medical thoracoscopy}

Before medical thoracoscopy, ultrasound was performed to confirm the optimal incision location (generally at midaxillary line between the seventh to eighth intercostal spaces). Local anesthesia with small dose of sufentanil and midazolam was given. A 1-to 2-cm incision was made. Then a flexible trocar was inserted, through which a semi-rigid thoracoscopy was inserted and connected to video. After aspiration of pleural fluid, the pleural space was carefully inspected. Suspicious pleural tissue was biopsied with biopsy forceps and sent for pathology examination.

\subsection{Diagnosis of tuberculous pleurisy}

Diagnosis of TPE was made if: caseous necrotic granulomas were found in pleural tissue; Ziehl-Neelsen stain or Lowenstein culture of pleural effusion fluid or pleural tissue, or Ziehl-Neelsen stain or Lowenstein culture of sputum were positive if the pleural effusion was accompanied.

\subsection{ADA and IGRA test}

ADA was measured in pleural fluid by colorimetric method of Guisti and Galanti (Bergmeyer \& Gawehn, 1974) using a commercial ADA kit (Adicon, Shenzhen, China). The concentration of interferon- $\gamma$ in blood sample was measured by enzyme-linked immunosorbent assay using commerical T-cell detection kit (Beijing wantai biopharmaceutical co., Ltd., China).

\subsection{Combination test}

To test whether combining the medical thorascopy, pleural fluid ADA and blood IGRA test together would improve diagnosis of TPE, we combined all the three tests together. The combination test flow chart was shown in Figure 1. Briefly, medical thorascopy, pleural fluid ADA and blood IGRA test will be performed, and a patient would be treated as TPE positive is any of the above tests is positive.

\subsection{Statistical analysis}

Continuous variables are presented as mean \pm standard deviation, while categorical variable are presented as numbers and percentages. Characteristics were compared using Student $\mathrm{t}$-test for continuous variables and Chi-square test for categorical variables. Receiver Operating Characteristic (ROC) curves were used to determine a cutoff value for tests. The optimum cutoff point was considered if the value provided the greatest sum of sensitivity plus specificity. The area under the curve (AUC) was used as a measure of test. AUC, sensitivity, specificity, positive likelihood ratios (PLR), and negative likelihood ratios (NLR) were calculated for individual test and combination test. $P$ values $<0.05$ was considered statistically significant. Data were analyzed using SPSS software version 19.0 (SPSS, Chicago, IL).

\section{Results}

\subsection{Patient characteristics}

A total of 154 patients with undiagnosed pleural effusion measured by chest X-ray or ultrasound were enrolled. Among them, 98 patients (63.6\%) were diagnosed as TPE in accordingly to our criteria. 56 patients (36.4\%) were diagnosed as non-tuberculous pleurisy. The mean age in TPE+ patients was $49.3 \pm 17.6$ years

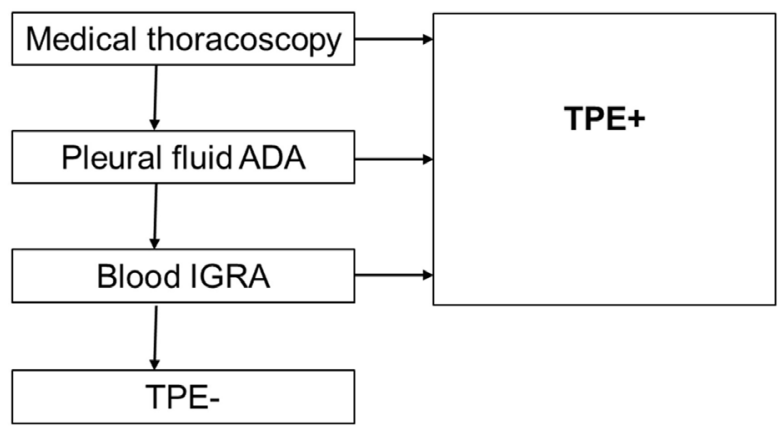

Figure 1. Flow chart of combination test. ADA: adenosine deaminase; IGRA: interferon-gamma release assay; TPE: Tuberculous pleural effusion. 
and in TPE- patients were $58.8 \pm 13.2$ years $(\mathrm{p}<0.05)$. In TPE+ group, 77 (78.6\%) were male. While in TPE- group, $39(69.6 \%)$ were male $(\mathrm{p}=0.30)$.

\subsection{Distributions of the biomarkers}

The distributions of the biomarkers in the two groups were summarized in Table 1. The mean pleural fluid ADA level was $41.6 \pm 15.0 \mathrm{U} / \mathrm{L}$ in the TPE+ group and $16.8 \pm 26.4 \mathrm{U} / \mathrm{L}$ in the TPE- group $(\mathrm{p}<0.001)$. Similarly, IGRA level was significant higher in the TPE+ group than the TPE- group $(111.2 \pm 155.6 \mathrm{pg} / \mathrm{mL}$ versus $20.1 \pm 63.7 \mathrm{pg} / \mathrm{mL}, \mathrm{p}<0.001)$. Seventy-four cases $(75.5 \%)$ were diagnosed as positive based on thoracoscopy pathology results in the TPE+ group, while no patient was diagnosed as positive in the TPE- group.

\subsection{Diagnostic performance}

The diagnostic thresholds obtained via receiver operating characteristic curve analysis were: ADA 23.4 U/L with AUC 0.91 (95\% CI: 0.85-0.97, Figure 2) and IFN- $\gamma 6.9 \mathrm{pg} / \mathrm{mL}$ with AUC 0.87 (95\% CI: 0.82-0.93). The AUC of medical thorascopy was 0.88 (95\% CI: 0.84-0.92). The performance of each diagnostic test was shown in Table 2. Pleural fluid ADA test had a sensitivity of 0.89 , specificity of 0.89 , PLR of 8.29 and NLR of 0.18 . Compared to ADA, blood IGRA has similar sensitivity (0.87), lower specificity (0.77), lower PLR (3.74), and higher NLR (0.23). Medical thorascopy has perfect specificity and sensitivity of 0.76 . By combining all three test together, we achieved sensitivity of 0.92 , perfect specificity and NLR of 0.082 . The AUC of the combination test was $0.96(0.93-0.99)$, which was significant higher than any individual test $(\mathrm{p}<0.001)$.

\section{Discussion}

Our study showed that although medical thorascopy, pleural fluid ADA, and blood IGRA each had good diagnostic performance, combining all three tests together significantly improve diagnostic performance of TPE. Based on our experience, the combining test is a practical test that could be applied clinically.

Despite the progress in the diagnosis and treatment of TB, TB is still a major health problem worldwide, particularly in developing countries (Abegunde et al., 2007). Lack of rapid, accurate, affordable, point-of-care TB diagnostic tests is a major barrier to control TB (Zumla et al., 2013). The gold standard diagnosis of TB is based on $\mathrm{M}$. tuberculosis isolation or observation of AFB in sputum examination, however, other diagnostic tests with shorter duration, good sensitivity and specificity are needed. As observed in our study, soluble biomarkers including ADA and IGRA test have good diagnostic performance. However, combining medical thorascopy with biomarkers test further improved the diagnostic performance and should be recommended.

In addition, literatures have shown that the combined test, especially medical thoracoscopy is safe and cost-effective. Studies have shown that medical thoracoscopy is a safe procedure in the diagnosis of exudative pleural effusion (Sakuraba et al, 2006; Wang et al., 2015a). Wang et al showed that no mortality has been associated with medical thoracoscopy, and the rates of major and minor complications are $1.5 \%$ and $10.5 \%$, respectively (Wang et al., 2015a). Although the aforementioned biomarkers have good sensitivity and specificity, neither of them provided definite etiologic diagnosis. In resource-poor areas with high incidence of tuberculosis, blind pleural biopsy is recommended as the first line management of pleural effusion (Hooper et al., 2010). Image-guided pleural biopsies, including ultrasound- or CT-guided pleural biopsies have been reported to be superior to blind pleural biopsies in diagnosis of malignant pleural effusion (Maskell et al., 2003). Not much comparison between image-guided pleural biopsies and medical thoracoscopy has been done. Metintas et al. (2010) showed that in patients with only pleural fluid appearance on CT scan and in those who may have benign pleural pathologies other than TB, medical thoracoscopy had higher sensitivity (94.1\% versus $87.5 \%)$ and is recommended. In our study, medical thoracoscopy had very good diagnostic performance. Additionally, Wang et al. showed that medical thoracoscopy is a cost-effective tool: its cost was similar to routine examinations involving biochemistry and cytology, sputum cytology, and thoracentesis (Wang et al., 2008). Another advantage of medical thoracoscopy compared to biomarkers is that medical thoracoscopy can be an effective

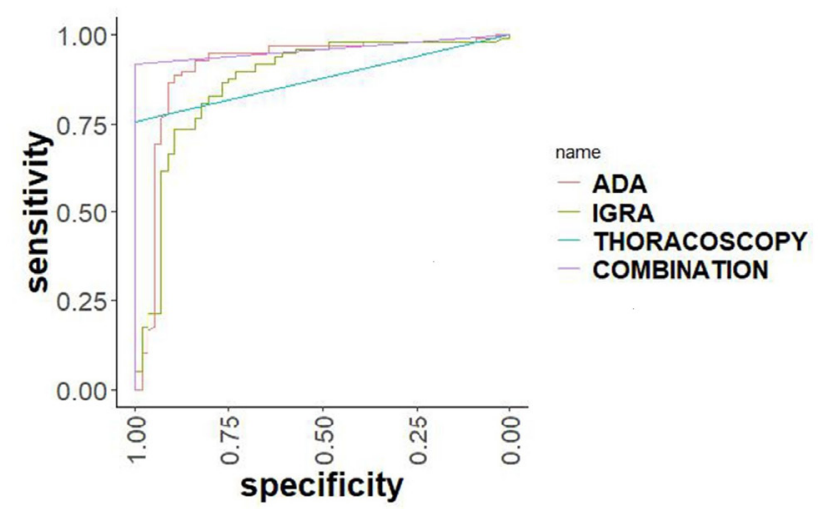

Figure 2. Receiver operating characteristic curves for diagnosis of tuberculous pleural effusion.

Table 1. Distribution of test results.

\begin{tabular}{lccc}
\hline & TPE- & TPE+ & P value \\
\hline ADA, U/L & $16.8(26.4)$ & $41.6(15.0)$ & $<0.001$ \\
IGRA, pg/Ml & $20.1(63.7)$ & $111.2(155.5)$ & $<0.001$ \\
Thoracoscopy positive & 0 & $74(75.5)$ & $<0.001$ \\
\hline
\end{tabular}

ADA: adenosine deaminase; IGRA: interferon-gamma release assay; TPE: tuberculous pleural effusion. P values $<0.05$ was considered statistically significant. 
Table 2. Performance of each diagnostic test.

\begin{tabular}{cllll}
\hline Test & Sensitivity & Specificity & PLR & NLR \\
\hline ADA & $0.89(0.80-0.94)$ & $0.89(0.77-0.96)$ & $8.29(3.88-17.71)$ & $0.18(0.098-0.30)$ \\
IGRA & $0.87(0.78-0.93)$ & $0.77(0.63-0.87)$ & $3.74(2.31-6.05)$ & $0.23(0.13-0.37)$ \\
MT & $0.76(0.66-0.83)$ & $1.0(0.92-1.0)$ & infinity & $0.25(0.17-0.35)$ \\
Combination & $0.92(0.84-0.96)$ & $1.0(0.92-1.0)$ & Infinity & $0.082(0.042-0.16)$ \\
\hline
\end{tabular}

ADA: adenosine deaminase; IGRA: interferon-gamma release assay; PLR: positive likelihood ratios; MT: medical thoracoscopy; NLR: negative likelihood ratios.

approach in the treatment of TPE, especially multiloculated and organized TPE (Xiong et al., 2016).

As mentioned, finding rapid, accurate, affordable, and point-of-care TB diagnostic tests is an essential part of TB control. Special challenges was emerging in immunosuppressed patients, such as patients with HIV. HIV-mediated immunosuppression impairs granuloma formation, and commonly results in frequent extrapulomary diseases including TPE, atypical chest radiographic findings, and lower concentration of bacteria (Perkins \& Cunningham, 2007), altogether results in special challenges in diagnosis. Our combination test, with high sensitivity and specificity, might be valuable in these high-risk populations with diagnosis challenges. Future studies are needed to validate our combination test in more specific population.

In summary, combining medical thoracoscopy with Interferon-Gamma release assay and adenosine deaminase performs better than individual test in diagnosis of TPE. The combination diagnosis has very high diagnostic rate, can be easily and safely carried out. Future studies are needed to further validate the performance in specific population.

\section{References}

Abegunde, D. O., Mathers, C. D., Adam, T., Ortegon, M., \& Strong, K. (2007). The burden and costs of chronic diseases in low-income and middle-income countries. Lancet, 370(9603), 1929-1938. http:// dx.doi.org/10.1016/S0140-6736(07)61696-1. PMid:18063029.

Bergmeyer, H.U., Gawehn, K. (1974). Methods of enzymatic analysis. Cambridge: Academic Press.

Gopi, A., Madhavan, S. M., Sharma, S. K., \& Sahn, S. A. (2007). Diagnosis and treatment of tuberculous pleural effusion in 2006. Chest, 131(3), 880-889. http://dx.doi.org/10.1378/chest.06-2063. PMid:17356108.

Hooper, C., Lee, Y. C., \& Maskell, N., \& BTS Pleural Guideline Group (2010). Investigation of a unilateral pleural effusion in adults: British Thoracic Society Pleural Disease Guideline 2010. Thorax, 65(Suppl 2), ii4-ii17. http://dx.doi.org/10.1136/thx.2010.136978. PMid:20696692.

Jelastopulu, E., Alexopoulos, E. C., Venieri, D., Tsiros, G., Komninou, G., Constantinidis, T. C., \& Chrysanthopoulos, K. (2009). Substantial underreporting of tuberculosis in West Greece: implications for local and national surveillance. Euro surveillance : bulletin Européen sur les maladies transmissibles = European communicable disease bulletin, 14(11), 19152. PMID: 19317978.

Light, R. W. (2010). Update on tuberculous pleural effusion. Respirology (Carlton, Vic.), 15(3), 451-458. http://dx.doi.org/10.1111/j.14401843.2010.01723.x. PMid:20345583.
Maskell, N. A., Gleeson, F. V., \& Davies, R. J. (2003). Standard pleural biopsy versus CT-guided cutting-needle biopsy for diagnosis of malignant disease in pleural effusions: a randomised controlled trial. Lancet, 361(9366), 1326-1330. http://dx.doi.org/10.1016/ S0140-6736(03)13079-6. PMid:12711467.

Mehta, J. B., Dutt, A., Harvill, L., \& Mathews, K. M. (1991). Epidemiology of extrapulmonary tuberculosis. A comparative analysis with pre-AIDS era. Chest, 99(5), 1134-1138. http://dx.doi.org/10.1378/ chest.99.5.1134. PMid:2019168.

Metintas, M., Ak, G., Dundar, E., Yildirim, H., Ozkan, R., Kurt, E., Erginel, S., Alatas, F., \& Metintas, S. (2010). Medical thoracoscopy vs CT scan-guided Abrams pleural needle biopsy for diagnosis of patients with pleural effusions: a randomized, controlled trial. Chest, 137(6), 1362-1368. http://dx.doi.org/10.1378/chest.09-0884. PMid:20154079.

Mlika-Cabanne, N., Brauner, M., Kamanfu, G., Grenier, P., Nikoyagize, E., Aubry, P., Larouzé, B., \& Murray, J. F. (1995). Radiographic abnormalities in tuberculosis and risk of coexisting human immunodeficiency virus infection. Methods and preliminary results from Bujumbura, Burundi. American Journal of Respiratory and Critical Care Medicine, 152(2), 794-799. http://dx.doi.org/10.1164/ ajrccm.152.2.7633744. PMid:7633744.

Pareek, M., Greenaway, C., Noori, T., Munoz, J., \& Zenner, D. (2016). The impact of migration on tuberculosis epidemiology and control in high-income countries: a review. BMC Medicine, 14, 48. http:// dx.doi.org/10.1186/s12916-016-0595-5. PMid:27004556.

Patiala, J. (1954). Initial tuberculous pleuritis in the Finnish armed forces in 1939-1945 with special reference to eventual postpleuritic tuberculosis. Acta tuberculosea Scandinavica. Supplementum, 36, 1-57. PMid:14376155.

Perkins, M. D., \& Cunningham, J. (2007). Facing the crisis: improving the diagnosis of tuberculosis in the HIV era. The Journal of Infectious Diseases, 196(Suppl 1), S15-S27. http://dx.doi.org/10.1086/518656. PMid:17624822.

Porcel, J. M., Esquerda, A., \& Bielsa, S. (2010). Diagnostic performance of adenosine deaminase activity in pleural fluid: a single-center experience with over 2100 consecutive patients. European Journal of Internal Medicine, 21(5), 419-423. http://dx.doi.org/10.1016/j. ejim.2010.03.011. PMid:20816597.

Saks, A. M., \& Posner, R. (1992). Tuberculosis in HIV positive patients in South Africa: a comparative radiological study with HIV negative patients. Clinical Radiology, 46(6), 387-390. http://dx.doi.org/10.1016/ S0009-9260(05)80684-1. PMid:1493651.

Sakuraba, M., Masuda, K., Hebisawa, A., Sagara, Y., \& Komatsu, H. (2006). Diagnostic value of thoracoscopic pleural biopsy for pleurisy under local anaesthesia. ANZ Journal of Surgery, 76(8), 722-724. http://dx.doi.org/10.1111/j.1445-2197.2006.03839.x. PMid:16916393.

Salmanzadeh, S., Tavakkol, H., Bavieh, K., \& Alavi, S. M. (2015). Diagnostic Value of Serum Adenosine Deaminase (ADA) level for pulmonary tuberculosis. Jundishapur Journal of Microbiology, 8(3), e21760. http://dx.doi.org/10.5812/jjm.21760. PMid:25861440. 
Trajman, A., Pai, M., Dheda, K., van Zyl Smit, R., Zwerling, A. A., Joshi, R., Kalantri, S., Daley, P., \& Menzies, D. (2008). Novel tests for diagnosing tuberculous pleural effusion: what works and what does not? The European Respiratory Journal, 31(5), 1098-1106. http:// dx.doi.org/10.1183/09031936.00147507. PMid:18448504.

Uskul, B., Turker, H., Ulman, C., Ertugrul, M., Selvi, A., Kant, A., Arslan, S., \& Ozgel, M. (2005). The relation of the pleural thickening in tuberculosis pleurisy with the activity of adenosine deaminase. Archivio Monaldi per le Malattie del Torace, 63(2), 101-107. PMid:16128225.

Wang, Z., Tong, Z. H., Li, H. J., Zhao, T. T., Li, X. Y., Xu, L. L., Luo, J., Jin, M. L., Li, R. S., \& Wang, C. (2008). Semi-rigid thoracoscopy for undiagnosed exudative pleural effusions: a comparative study. Chinese Medical Journal, 121(15), 1384-1389. http://dx.doi. org/10.1097/00029330-200808010-00010. PMid:18959114.

Wang, X. J., Yang, Y., Wang, Z., Xu, L. L., Wu, Y. B., Zhang, J., Tong, Z. H., \& Shi, H. Z. (2015a). Efficacy and safety of diagnostic thoracoscopy in undiagnosed pleural effusions. Respiration; International Review of Thoracic Diseases, 90(3), 251-255.
Wang, Z., Xu, L. L., Wu, Y. B., Wang, X. J., Yang, Y., Zhang, J., Tong, Z. H., \& Shi, H. Z. (2015b). Diagnostic value and safety of medical thoracoscopy in tuberculous pleural effusion. Respiratory Medicine, 109(9), 1188-1192. http://dx.doi.org/10.1016/j.rmed.2015.06.008. PMid:26166016.

World Health Organization - WHO. (1993). WHO declares tuberculosis a global emergency. Sozial- und Praventivmedizin, 38(4), 251-252. PMID: 8212917.

World Health Organization - WHO. (2013). World Health Organization Global Tuberculosis Report 2013. Geneva: WHO. Retrieved from http:// apps. who. int/iris/bitstream/10665/91355/1/9789241564656_eng.pdf

Xiong, Y., Gao, X., Zhu, H., Ding, C., \& Wang, J. (2016). Role of medical thoracoscopy in the treatment of tuberculous pleural effusion. Journal of Thoracic Disease, 8(1), 52-60. PMid:26904212.

Zhai, K., Lu, Y., \& Shi, H. Z. (2016). Tuberculous pleural effusion. Journal of Thoracic Disease, 8(7), E486-E494. http://dx.doi.org/10.21037/ jtd.2016.05.87. PMid:27499981.

Zumla, A., Kim, P., Maeurer, M., \& Schito, M. (2013). Zero deaths from tuberculosis: progress, reality, and hope. The Lancet. Infectious Diseases, 13(4), 285-287.http://dx.doi.org/10.1016/S1473-3099(13)70039-2. PMid:23531385. 